28 | 2002

Histoire de l'enseignement du français langue

étrangère ou seconde dans le bassin méditerranéen.

Volume 2

\title{
L'enseignement de la prononciation du français en Espagne au XIXe siècle
}

\section{Maria Eugenia Fernández Fraile}

\section{(2) OpenEdition \\ Journals}

Édition électronique

URL : https://journals.openedition.org/dhfles/2633

DOI : $10.4000 /$ dhfles.2633

ISSN : 2221-4038

Éditeur

Société Internationale pour l'Histoire du Français Langue Étrangère ou Seconde

Édition imprimée

Date de publication : 1 juin 2002

Pagination : $33-51$

ISSN : 0992-7654

Référence électronique

Maria Eugenia Fernández Fraile, «L'enseignement de la prononciation du français en Espagne au XIXe siècle », Documents pour l'histoire du français langue étrangère ou seconde [En ligne], 28 | 2002, mis en ligne le 31 janvier 2014, consulté le 27 mai 2021. URL : http://journals.openedition.org/dhfles/2633 ; DOI : https://doi.org/10.4000/dhfles.2633

Ce document a été généré automatiquement le 27 mai 2021.

(c) SIHFLES 


\title{
L'enseignement de la prononciation $\mathrm{du}$ français en Espagne au XIXe siècle
}

\author{
Maria Eugenia Fernández Fraile
}

1 L'enseignement de la prononciation du français en Espagne a fait l'objet d'une série d'études qui concernent surtout les XVI ${ }^{e}$, XVII e et XVIII e siècles (D. Fischer, M. Bruña, A. Supiot ${ }^{1}$...). Notre article se situe dans la continuation de ces études. Tomás Escriche y Mieg $(1882)^{2}$ classe au nombre de trois les approches sur l'enseignement de la prononciation au cours du XIX ${ }^{\mathrm{e}}$ siècle :

2 - certains auteurs " se limitent à suivre un plan purement théorique ${ }^{3}$ » : ils reprennent donc l'essentiel de la méthode traditionnelle théorique (MT), que nous trouvons, entre autres, dans les manuels de Tramarría (1829), Dupuy (1838), Thiers (1841), Cornellas (1845), Sauzeau (1845), Bergnes de las Casas (1845), Ascaso y Pérez (1876).

3 - « d'autres se décident en faveur d'une méthode théorico-pratique » (MTP): cette méthode, établie par Chantreau, est reprise selon divers degrés et modalités d'intégration entre théorie (règles, explications) et pratique : c'est le cas, entre autres, de Bouynot (1815), de Delaborde (1859), de Trauque (1863), de Borde (1888), de Coste (1892), ou de Reinaud (s.d.).

4 - un dernier groupe d'auteurs-professeurs " préfèrent un enseignement pratique ": ceux-ci vont proposer une approche méthodologique où la pratique joue un rôle essentiel. La théorie y est totalement absente : il s'agit surtout des adaptations espagnoles de la méthode de Ahn (Mac-Veigh, 1890 ; Atalo Castañs, s.d.), mais aussi d'autres propositions comme celle de Pou y Clara (1886).

5 Cependant, dans d'autres cas, la pratique devance mais n'élimine pas la présentation des règles ; nous pouvons ainsi parler d'une quatrième modalité : la méthode praticothéorique (MPT), qui conçoit des procédés totalement nouveaux d'enseignement de la prononciation : c'est le cas de Piferrer $(1843,1846)$, ou de Mendizábal (1860). Nous allons présenter un bref aperçu de ces différentes approches, en regroupant dans notre exposé les méthodes théorique et théorico-pratique en un seul chapitre. 


\section{Les méthodes théorique (MT) et théorico-pratique (MTP) d'enseignement de la prononciation}

6 La démarche théorique est suffisamment connue pour ne pas nous y arrêter longtemps : il suffit de reprendre la caractérisation générale proposée par M. J.Salema (1997 : 19, 117), qui est valable pour les grammaires du FLE édité en Espagne : il s'agit en effet d'un « apprentissage essentiellement théorique, basé surla mémorisation des règles de la prononciation et sur la description des sonsconfondus avec les lettres. " L'objectif consiste bien sûr à faire que les élèves parviennent à lire correctement des textes écrits : suppose qu'une lecture correcte (c'est-à-dire conforme aux règles exposées) est la clé d'une bonne prononciation. Il va de soi que cette lecture se faisait toujours à voix haute. Le contenu théorique qui doit être mémorisé par les élèves consiste ainsi en une description, sous forme de règles (interminables) entre la graphie (d'où l'on part) et les sons. On ne cache pas que la " connaissance d'une langue n'est possible que par de longs efforts » (Sauzeau 1845 : Prologue). Quant à la méthode théorico-pratique (MTP), elle possède une série de caractéristiques qui l'éloignent de la MT : la présentation des règles n'est pas faite en un seul bloc théorique, mais on fait place, à la suite de chaque règle ou petit groupe de règles, à des exercices de prononciation ou de lecture rattachés aux règles exposées. Dans la $\mathrm{MT}$, par contre, la phase de mémorisation (parfois, de longs recueils de règles) précédait obligatoirement le passage à l'application (lecture), qui s'effectuait sur des textes non spécialement conçus dans le but de faire appliquer des règles spécifiques. Les différences entre MT et MTP concernent donc surtout l'approche méthodologique, et dans un moindre degré le contenu grammatical concernant la prononciation (les "règles »), qui est similaire. On peut toutefois reconnaître une tendance vers la simplification des règles dans la MTP, comme conséquence de cette volonté de consacrer une plus grande place à l'application.

7 La prononciation occupe la première partie des grammaires (ou l'Introduction) : c'est le cas des manuels de Bouynot (1815), Tramarria (1829), Cornelias (1844), ou Sauzeau (1845). Mais aussi, ce qui montre l'intérêt croissant pour les questions phonétiques tout au long du siècle, on rédige des ouvrages spécifiques : c'est le cas de Thiers (1841), de Ascaso y Pérez (1854), de Trauque (1863), de Borde (1888), ou de Coste (1892). Quant à Escriche y Mieg, il publie aussi un ouvrage spécialement destiné à la pratique (1881), puisqu'il considère que son ouvrage antérieur (Prontuario de pronunciación francesa ${ }^{4}$ ) est incomplet sans cet « ensemble systématique d'exercices » $(1881: \mathrm{V})$.

8 A partir du milieu du siècle, certains auteurs transforment le mode de présentation du contenu ; ils abandonnent la division en parties thématiques (voyelles, consonnes, prosodie...) au profit de leçons plus courtes : Thiers (1841) établit 6 leçons (théorie uniquement, 32 pages); Trauque (1863), 10 leçons (19 pages, avec les textes d'application); Borde (1888), 20 leçons (73 pages avec les exercices d'application); Coste (1892), 34 leçons (46 pages, avec les textes d'application)...

Quant au contenu lui-même, on retrouve dans ces descriptions, en gros, le même contenu que celui des grammaires françaises éditées en France à l'époque ; de fait on peut dire que les auteurs espagnols suivent de près le peu d'innovations produites en France (jusqu'à l'irruption des phonéticiens). Ainsi, on continue généralement de dire que les voyelles sont au nombre de six $(a, e, i, o, u, y)$ et qu'on les appelle voyelles " parce qu'elles seules peuvent former une voix, un son »; on parle de voyelles brèves (son 
simple, comme dans patte) et longues (comme dans mâle) : Dans la langue française, les voyelles brèves sont toujours aiguës, et les graves sont toujours longues » (GiraultDuvivier 1830 : 7); quant aux consonnes, on conserve encore la vieille définition latine : " on les appelle consonnes parce qu'elles ne peuvent exprimer un son qu'avec le secours des voyelles " (Noël, Chapsal 1849: $1^{5}$ ). C'est le cas aussi des grammaires éditées en Espagne : Bergnes (1845), Sauzeau (1845), Piferrer (1846), Ascaso (1854), Reinaud (s.d.), etc. Certains continuent même de distinguer les consonnes doubles ou composées ch, ph, th, rh, ll, gn (Thiers $1841: 11)$. Tramarría (1829 : 9), en parlant des éléments prosodiques du français, distingue l'accent (l'intensité, ou élévation de la voix quand on prononce une syllabe), l'aspiration (l'effort ou l'impulsion qu'on produit sur le son), la quantité (syllabes brèves, prononcées en un temps ; syllabes longues, prononcées en deux temps; syllabes communes ou douteuses, qui peuvent être longues ou brèves selon les cas).

Aussi, même si l'on part de l'écrit dans les descriptions, certains grammairiens français se rendent compte qu'il existe une différenciation entre la lettre écrite et le son : les voyelles indiquées (ou caractères écrits, au nombre de 6), possèdent des 'inflexions' (des sons différents) de la voix. Sophie Dupuis se montre contraire à une différenciation des voyelles en longues et en brèves en faveur d'une distinction selon le son (in Millet 1933 : 84). Elle distingue ainsi 18 voyelles, dont 5 nasales. N. Landais établit de son côté l'existence de 2 a, 4 e, 2 o, $2 \mathrm{eu}, 1 i, 1 \mathfrak{u}, 1 \mathrm{ou}, 4$ nasales, soit, avec l'e muet, 18 sonsdifférents (in Millet $1933: 84$ ). Littré propose 19 « voyelles » ou sonsdifférents : 2 a (long, bref), $4 e$ (muet, fermé, ouvert, long très ouvert), $2 i$ (long, bref), 20 (aigu, grave), 1 ou, appelé diphtongue, $2 u$ (long, bref), 2 oe (aigu, grave), 4 nasales (in Millet 1933: 85). Quant à Girault-Duvivier (1830:7),suivant les propositions de « plusieurs grammairiens ", il propose d'éliminerles réalisations graves et aiguës des voyelles, et de limiter le nombre devoyelles à $13: 1 a, 3 e$ (ouvert, fermé, muet), $1 i, 1 \mathrm{o}, 1 \mathrm{u}, 1 \mathrm{eu}, 1 \mathrm{ou}, 4$ nasales. Ces présentations constituent, comme l'indique A. Millet, des " indices d'un fléchissement de l'intérêt porté aux études des sons » (1933 : 86).

11 On retrouve ces classements, avec des variantes, dans certaines grammaires pour l'enseignement du français éditées en Espagne. Par exemple, les tableaux suivants, de Sauzeau $(1845: 1)$ et de Thiers $(1841: 1)$ sont assez proches des descriptions françaises de l'époque :

\begin{tabular}{|c|c|}
\hline $\begin{array}{l}\text { Sauzeau } \\
\text { Cuadro de las diferentes } \\
\text { especies de vocales simples }\end{array}$ & $\begin{array}{l}\text { Thiers } \\
\text { Tabla de las vocales simples }\end{array}$ \\
\hline 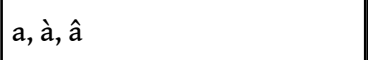 & a e i o u y \\
\hline e, é, è, ê, ë & é \\
\hline $\mathrm{i}, \hat{\mathrm{i}}, \mathrm{i}$ & à è \\
\hline $0, \hat{o}$ & $\hat{a} \hat{e} \hat{i} \hat{o} \quad \hat{u}$ \\
\hline $\mathrm{u}, \mathrm{ù}, \hat{u}, \ddot{u}$ & $\ddot{e} \quad \ddot{i} \quad \ddot{u}$ \\
\hline 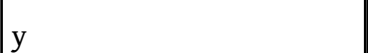 & \\
\hline
\end{tabular}


Sauzeau et Thiers distinguent ainsi trois types de prononciation dans la voyelle $a$ ( $a$ et $\grave{a}$ se prononcent comme en espagnol : place; $a$ est long dans certains mots comme phase ; $\hat{a}$ est grave et long : pâte), ou bien 5 espèces de voyelle $e$ (ou même 6 dans le cas de Sauzeau, puisqu'il fait la différence entre le «è grave » de père et le «è ouvert » de accès, 1845 : 2). Les explications sur les sons reprennent les critères des grammaires françaises de l'époque, à savoir : - on part des lettres, pour exposer leurs différentes réalisations phoniques ;

14 - on adopte principalement le critère acoustique pour définir les sons, et les appellations reprennent ce point de vue : le $e$ muet français est « doux et sourd, et il faut faire un léger effort pour le faire sortir des poumons » (Coste $1892: 11^{6}$ ) ; les sons nasaux sont « gangosos » (nasillards), semblables au son du métal (Thiers $1841: 19$ ), etc.;

15 - la description articulatoire n'est pas systématique, et ne tient compte que de la position de la langue ; l'arrondissement des lèvres est vu sous l'angle de l'effet acoustique produit (son grave) et n'est pas associé à la position de la langue ;

16 - bien sûr, comme élément spécifique, les manuels espagnols ajoutent l'appel fréquent à la comparaison avec l'espagnol, voire le catalan ou l'andalou, ce qui leur permet d'éliminer de nombreuses explications.

17 On trouve aussi de nombreuses observations et réflexions sur la sélection du contenu phonétique à inclure dans la grammaire, sa place, son rôle dans l'apprentissage, ou encore sur la façon de procéder pour l'enseignement de la prononciation :

18 a) la première concerne la description du contenu phonétique. Vers la fin du siècle, les grammaires (manuels, traités) éditées en Espagne commencent à se faire l'écho des recherches linguistiques effectuées en France (filiation des langues, linguistique historique, comparatisme, dialectologie), recherches qui devront entraîner, selon Escriche y Mieg, une profonde modification des « connaissances qui doivent être transmises à l'élève ${ }^{7}$ " $(1882: 140,278)$. A partir de la linguistique historique, les grammaires pourront cesser d'être des ensembles de règles capricieuses puisque, tout en remontant aux sources philologiques, on peut découvrir sous les règles les lois générales auxquelles la langue obéit, et donc réduire le nombre de règles. D'autre part, grâce au comparatisme, on pourra éliminer de la présentation grammaticale tout ce qui est commun avec la langue maternelle de l'élève $(1882: 140,278)^{8}$.

19 Escriche y Mieg incorpore aussi - dans ses Ejercicios linguales (1881) - certaines descriptions des phonéticiens, et déclare " n'avoir pris en compte que les sons, et nullement les lettres, étant donné que son objet est strictement phonétique ${ }^{9}$ » (1881 : IX). Il adopte une approche physiologique dans les explications, et abandonne les traditionnelles définitions acoustiques : " je n'ai point cru inutile de donner, pour chaque son et chaque articulation inconnue, une courte explication des organes qui y prennent part, ainsi que de la disposition qu'ils adoptent ${ }^{10} »$ (1881: IX). Par exemple, il n'établit aucune différence entre le son du e muet (" semi-muet », selon lui) et celui de $e u$ : « ce son, qui est facile à produire, tient de la nature du e et du o espagnols. Du premier son, il prend la position de la langue, et, du deuxième, il prend celle des lèvres. Pour prononcer cette voyelle, il suffit de placer la langue comme si on allait prononcer un e, et lancer l'air, en avançant un peu les lèvres et en les arrondissant comme si on allait produire uno ${ }^{11} »(1881: 14-15)$. Il décrit avec exactitude l'articulation du « u français»: «Cette voyelle tient de la nature du i et du u espagnols. Elle prend de la 
première la position de la langue et de la deuxième celle des lèvres. Pour émettre ce son, il suffit de placer la langue comme s'il s'agissait d'un i, et de mettre les lèvres comme si on voulait prononcer le u espagnol ${ }^{12} »(1881: 17)$.

b) la simplification des règles et le besoin de se baser sur la pratique, selon le principe : "Peu de règles, et beaucoup de pratique " (Bouynot 1815 : 15). Bouynot laisse au professeur la tâche de fournir les explications sur les différents sons de "vive voix ${ }^{13}$ " (Bouynot 1815 : III). De nombreux auteurs suivront le long du siècle le chemin marqué par Bouynot : Trauque organise sa Clave de lectura (1863) en 10 leçons, qui suivent le schéma suivant : l'énoncé qui contient deux ou trois règles (5-7 lignes) est accompagné d'un long texte d'application (20-30 lignes, avec traduction interlinéaire). Les règles antérieures sont reprises dans la leçon suivante ; des notes en pied de page expliquent des questions particulières ; un index final $(1863: 28-29)$ classe les différentes écritures et renvoie aux pages correspondantes : l'effort de mémorisation des règles est pratiquement remplacé par le travail constant et lent de la pratique de la lecture, puisque le contenu présent dans chaque règle est très réduit (il introduit cependant pas moins de 108 notes en pied de page, qui constituent autant de règles ou d'exceptions). Borde (1888) propose une organisation du contenu très similaire à celle de Trauque : d'abord, de courtes indications sur la prononciation d'une graphie (par exemple : l'accent tonique, les consonnes finales, les types de e), puis des mots isolés (avec utilisation de typographies différentes pour indiquer les consonnes qui ne sont pas prononcées), enfin des phrases de langue courante (avec traduction interlinéaire des mots difficiles). La présentation du contenu phonétique chez Borde est d'autre part très original, et montre une expérience d'enseignement très longue et réfléchie : il le réduit aux principaux problèmes que rencontrent les Espagnols, et s'écarte résolument de la présentation des grammaires théoriques (il commence par exemple par la question de l'accent tonique et des consonnes finales). Escriche y Mieg (1881) propose de son côté des exercices syllabiques, des mots isolés, puis un choix de phrases (littéraires pour la plupart). Quant à Nevot (s.d.), il destine les 10 premières leçons à la prononciation, proposant une règle par leçon (par exemple, leçon 9 : ou se prononce comme le $u$ espagnol), qui est suivie d'une série de mots servant à pratiquer la correspondance écrit-oral proposée et à faire acquérir la règle (une page par leçon); la leçon 11 reprend sous forme de résumé les règles antérieures. La simplification du contenu théorique est, dans ce cas, énorme.

Nous allons finalement nous occuper d'un dernier auteur : Delaborde. Delaborde était docteur en médecine avant de se consacrer aux « Lettres. » Il reconnaît s'être basé pour composer sa " méthode théorique et pratique de langue française en 80 jours » (1859: Préface) sur les mêmes principes que Robertson ou Ollendorf. Sa méthode incorpore principalement le stratagème suivant : elle présente tout au début de son ouvrage un tableau de signes (des chiffrespour la plupart) qui correspondent aux sons du français. Le manuel de Delaborde présente aussi l'avantage d'inclure une introduction méthodologiquetrèsminutieuse, où il explique pas à pas sa méthode d'enseignement de la prononciation: le professeur lit le texte une première fois très lentement, puis une deuxièmefois ; à la troisième fois, l'élève répète mot à mot après lui, et celui-ci procèdeaux corrections, nécessaires ; finalement, l'élève lit le texte tout seul. A partirde la sixième leçon, deux lectures de la part du professeur sont suffisantes, puis, à partir de la trentième leçon, une seule lecture. 

importe, mais laréalisation des exercices. La méthode théorico-pratique, même dans le casextrême de Delaborde, ne débouche pas cependant sur la négation de la théorie. Il continue de penser l'apprentissage de la langue en situation scolaire en tantqu'appropriation pratique et conceptuelle; savoir une langue comporte deux pôles indissociables : un savoir-faire (lire, traduire, parler), mais aussi un savoir sur la langue (compréhension, explication des phénomènes, connaissance des règles de prononciation) tout aussi important.

c) Même à l'intérieur de la MT on envisage la « vive voix du maitre » comme élément nécessaire : les langues ont quelque chose d'irréductible, de particulier, qui constitue le « génie » de chacune d'elles, bien sûr dans le lexique, dans les constructions, mais aussi dans leurs qualités sonores (sons isolés, mélodie, etc.), qui ne peut pas être totalement expliqué (ce qui suppose une contradiction avec la mentalité rationnelle qui préside à la MT, comme l'indique Escriche y Mieg). De nombreuses descriptions de sons sont ainsi confiées à la prononciation du professeur.

\section{La méthode pratique}

Selon Escriche y Mieg, la méthode pratique partage avec la méthode théorico-pratique la croyance selon laquelle une langue n'obéit pas aux règles, mais que les règles sont tirées de la langue, et que par conséquent il faut apprendre la langue, et pas les règles. À son avis, c'est l'idée de base des " grammaires pratiques ", par exemple celles de Robertson, Ollendorf, Ahn, etc. Cependant, " ces méthodes oublient à leur tour que l'apprentissage de la langue maternelle est très lent, et qu'un étranger qui apprend tout seul d'autres langues n'arrive qu'à une connaissance très superficielle au bout d'un travail soutenu » $(1882: 277)^{14}$.

Dans ces "méthodes pratiques ", l'enseignement de la prononciation se base sur les principes de l'imprégnation naturelle (méthode maternelle) et de l'imitation de la vive voix du maître. Les adaptations espagnoles de Ahn -notamment, celle de H. Mac-Veigh (1890) et celle de A. Castañs (s.d.) - ne destinent à la prononciation qu'une Introduction de deux pages (recto-verso), destinée à fournir les correspondances minimales entre l'écrit et l'oral. La présence du maitre est par conséquent plus nécessaire que jamais :

Apprenez une langue étrangère de la même façon que vous avez appris votre langue maternelle : [...] C'est la méthode de la nature même et celle que toute mère utilise quand elle parle à son enfant, en lui répétant cent fois les mêmes mots, en les combinant de façon imperceptible, et en lui faisant parler la langue qu'elle parle ${ }^{15}$ (Mac Veigh 1890 : Préface).

Aucune explication articulatoire n'est fournie : toute la confiance est faite dans la capacité d'assimilation naturelle et de reproduction inconsciente propre des enfants.

Nous allons faire une brève référence à une adaptation " pratique » réalisée par J. Pou y Clara qui s'éloigne de la méthode Ahn. Le Método Pou est un système pratique, spécifiquement destiné à la prononciation :

Cette méthode est tout simplement de lecture française pratique, et par conséquent sans règles, parce qu'on n'en a pas besoin, si le professeur sait et veut accomplir son devoir ${ }^{16}(1886$ : Avertissement).

J. Pou adapte le procédé de Delaborde, c'est-à-dire un système de correspondances entre les différents sons du français et des codes numériques ; l'élève, au cours de sa 
lecture, voit ainsi immédiatement quel son il doit prononcer, et on évite ainsi l'énumération des règles nécessaires pour une reproduction orale (lecture) correcte d'un texte écrit. Ce système de correspondances numériques est intégré à une transcription phonique des textes, particulière à Pou, qui est présentée dans les pages de droite, à la vue de l'élève : une fois proposé le tableau de correspondances, et une demi-page d'exercices pour s'y habituer, le reste de l'ouvrage (vingt-trois pages) ne contient que les textes en orthographe courante (pages paires) et la transcription phonique correspondante (pages impaires).

\section{La méthode pratico-théorique}

Deux auteurs déclarent de façon explicite suivre une méthode pratico-théorique : Piferrer (1852) et Mendizábal (1860). Cette méthode inverse l'ordre traditionnel de présentation : les exercices devancent le contenu concernant la prononciation. Pour ce faire (c'est-à-dire, pour faire que l'élève lise un texte sans connaitre au préalable aucune règle ou correspondance), le rôle de l'imitation du maître devient primordial, comme dans la méthode pratique. Mais ces auteurs ont aussi recours à d'autres procédés. Piferrer incorpore une colonne qui reprend le texte de base sous forme de prononciation figurée : on reconnaît là bien sûr le système déjà utilisé en Espagne par Galmace au XVIII ${ }^{e}$ siècle. Le procédé de transcription phonétique complète des textes sera repris à la fin du siècle par les partisans de la méthode directe, en application des nouvelles recherches et propositions des phonéticiens, avec une différence toutefois : il ne s'agira pas d'un code approximatif qui restitue les sons étrangers sous le système graphique habituel de l'élève, mais d'un système de transcription basé sur des correspondances univoques entre son et signe graphique.

Dans la méthode de Piferrer, l'élève (tout seul, pouvant même se passer du maitre) commence par la lecture de la colonne de la prononciation (placée entre le texte français, à gauche, et la traduction, à droite), puis il lit le texte français, « en essayant de le lire exactement comme il est indiqué dans la colonne de la prononciation » (1852 : 8); à la fin de chaque leçon ( 13 au total) est présentée une portion de "théorie », qui reprend l'essentiel du contenu habituel concernant la prononciation. La phase pratique devance ainsi chronologiquement la présentation du contenu théorique, qui est incorporé dans les différentes leçons selon un ordre traditionnel, et sans rapport aux textes d'appui.

31 Quant à Joaquín Mendizábal, il fait un pas de plus : il associe directement pratique et théorie, tirant un parti très fort de cette inversion. Cet auteur, professeur titulaire au lycée de Saragosse, a adapté la méthode Robertson à l'Espagne avec sa Gramática francesa según el método de Mr. Robertson, « suivant les pas de l'auteur du système praticothéorique " (1860: Prologue), à partir de son expérience comme professeur qui l'a amené à un réaménagement complet de la première édition, qui date de 1856 . Le système de Mendizábal-Robertson se fonde sur deux piliers :

32 a) la simplification des règles de prononciation à l'aide d'un tableau montrant des correspondances numériques entre des sons et des chiffres, similaire à celui de Delaborde, dont l'utilisation est très aisée à acquérir. À la différence de Piferrer ou de Pou, qui présentent une transcription phonétique complète des textes, il reproduit les textes en graphie courante (pour ne pas créer des confusions d'orthographe ultérieures), en plaçant les codes numériques au-dessus des lettres ou des syllabes 
qu'ils veulent signaler, et sans traduction interlinéaire (voir documents en annexe pp. 50-51) : la phase de prononciation et celle de compréhension sont ainsi séparées (pour que toute l'attention de l'élève soit fixée sur l'écoute et la reproduction des sons). Étant donné que la prononciation est l'élément premier de toute méthode, la nouveauté de la démarche est évidente et tout de suite appréciée par les élèves ; lecture ${ }^{17}[\ldots](1860: \mathrm{V})$. questions sur le texte et réponses... des mots et de réflexion sur le fonctionnement de la langue. coup $^{19} »(1860: \mathrm{V})$, sans réfléchir sur les éléments qui le composent. convenablement : langue il est nécessaire de répéter et répéter à satiété ${ }^{20}(1860$ : IV).

- une phase pratique : tous les cours commencent par la lecture du texte numéroté :

on doit faire la lecture sur le texte numéroté ; c'est le professeur qui commence, qui lit tout le texte, et après lui cinq ou six élèves, ou bien davantage, jusqu'à ce que la classe entière soit imbibée par la prononciation. Pour que la sensation produite par l'accent du professeur ne faiblisse pas dans l'oüe des élèves, il convient fortement que le professeur lise le texte à nouveau chaque fois que deux élèves ont fait la

D'autres exercices viennent à la suite : version, thème, traduction orale (avec le livre fermé), traduction mot à mot, puis traduction de courtes phrases du texte ou composées avec les éléments du texte, formulation par le professeur de brèves

- une phase théorique : on reprend une série de mots isolés contenus dans letexte qu'on vient de travailler (par exemple : «allait - les - cuisiniers»), on propose une explication au sujet de ces mots (analyse : " tous lesmots antérieurs se terminent en consonne »), puis vient l'énoncé de larègle correspondante : « Règle 1 . La consonne finale est muette enfrançais. " Le contenu grammatical phonétique est ainsi décomposé le long des cinquante leçons du manuel, avec une progression solidement établie, selon un ordre totalement différent de celui des grammaires théoriques, avec un souci évident d'aller du plus simple au plus complexe, et en prenant compte des difficultés rencontrées par les élèves espagnols. On ne peut pas à proprement parler de méthode inductive (même s'il utilise cette dénomination) puisque les règles sont données et non pas découvertes par l'élève, mais on n'en est pas loin, puisque celui-ci est associé au travail d'analyse

Le « système pratico-théorique » de Mendizábal-Robertson - valable pour l'ensemble de l'apprentissage de la langue, et pas seulement pour la prononciation - se base donc sur l'imprégnation et l'imitation de la vive voix du maître, principes de la méthode maternelle, qui sont repris dans la première phase du travail : l'apprentissage d'une langue consiste en effet « non pas à apprendre des théories, mais à créer une habitude de parler à côté d'une autre habitude déjà présente, celle de la langue native [...] On doit s'en convaincre : on ne possède le français que lorsqu'on pense en français ${ }^{18}$ " (1860 : IV-V). Pour arriver à parler, on doit être capable d'émettre l'énoncé « tout d'un

Cependant, des distances sont prises par rapport à la méthode maternelle, et des exercices constants de répétition sont proposés pour aider ce principe à fonctionner

J'enjoins les élèves à ne pas passer à l'exercice suivant sans dominer l'exercice antérieur ; ils ne doivent pas avoir peur de se lasser de les répéter jusqu'à ce qu'ils soient convaincus de les dominer [...]. En effet, pour arriver à penser dans une

Des distances sont prises également par rapport à la méthode pratique, puisque, dans le système de Mendizábal-Robertson, les progrès de l'élève ne sont pas dus à une " 
répétition routinière», mais à " un dosage convenable entre la pratique et la théorie», et où « l'apprentissage, très simple, fait appel à la compréhension intelligente pour une bonne part du travail qui est confié àla mémoire dans d'autres systèmes ${ }^{21}$ " (1860 : IIIIV). Et finalement, le système de Mendizábal-Robertson prend d'énormes distances par rapport à la MT et la MTP, par l'inversion des phases :

au lieu d'un cumul de règles de prononciation, d'orthographe, d'analogie et de syntaxe, dont l'élève ne voit pas une application immédiate, et qui tendent constamment à s'échapper de sa mémoire [...], [ce système] lui offre dès le début une série d'exercices aussi faciles que simples ${ }^{22}$ (1860 : III-IV).

Comme conclusion, nous devons mettre en relief que les manuels d'enseignement du français du XIX siècle montrent une énorme richesse de propositions quant à la description du contenu phonétique, sa sélection, sa présentation, où l'on voit se manifester les innovations produites en France. Mais aussi, et surtout, des propositions nouvelles voient le jour de la part des auteurs espagnols eux-mêmes à partir de leur expérience professionnelle. Ces propositions se produisent surtout du côté de la méthodologie de l'enseignement de la prononciation, où le débat d'idées montre un constant souci de faire mieux qu'avant, et mieux que les autres : l'enseignement de la prononciation est bel et bien quelque chose de vivant, qui bouge, qui grésillede vie. De nombreuses voix s'élèvent en faveur d'un apprentissage pratique etpas simplement théorique de la prononciation, à travers des exercicesd'application plus nombreux ; d'autres soutiennent que c'est l'imprégnation à travers l'ouïe à partir de la vive voix du maître qui constitue le meilleur système, approche qui ouvre la voie dans les esprits en faveur de la méthode directe ; des positionnements extrêmes vont jusqu'à nier toute efficacitéà l'enseignement théorique : nous voyons que se produisaient déjà les mêmes débats théoriques que ceux qui se posent aujourd'hui, même si les moyens et l'appareillage technique utilisé n'étaient pas les nôtres, bien sûr. Voilà l'intérêt de se pencher sur ces auteurs-professeurs, puisqu'on y apprend que l'essentiel consiste à " savoir quoi faire ", et non pas à posséder des outils plus ou moins sophistiqués. 


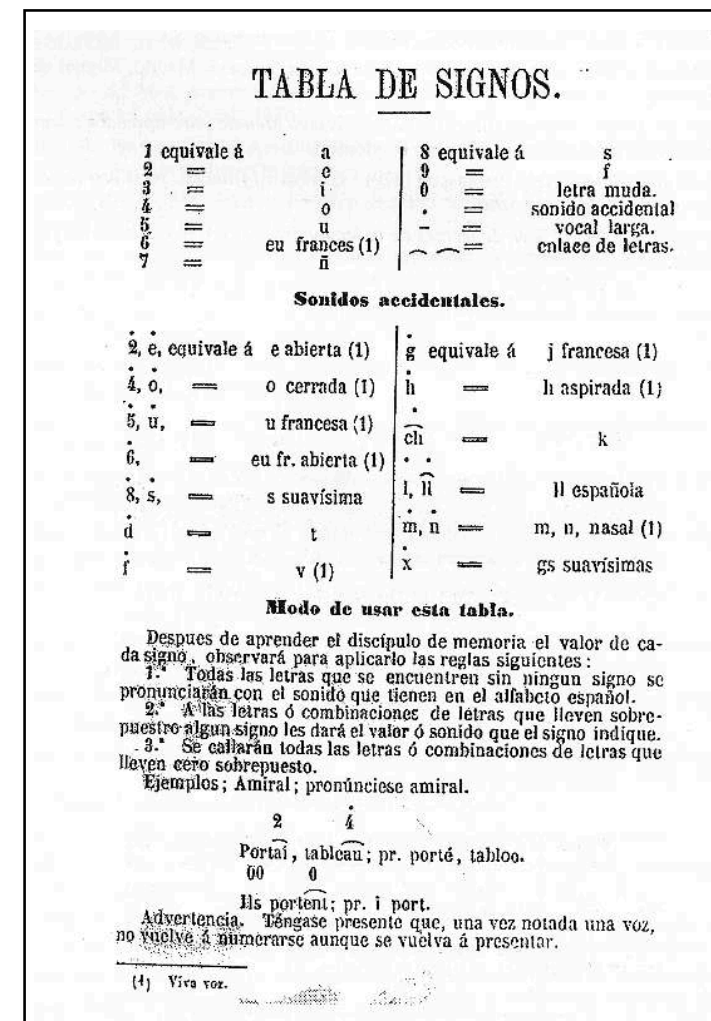

MENDIZÁBAL, Joaquín (1860)

Gramática francesa, $2^{\mathrm{e}}$ éd., Saragosse, Vicente Andrés

\section{$\underset{\text { Primera }}{\stackrel{6}{6} \text { Leccion. }}$}

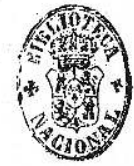

PRIMERA PARTÉ.-PRÁCTICA.

LECTURA.

$00 \quad 5 \quad 0 \quad 20 \quad 000$

Lorsque la cour allait à l' Escurial, les cui-

- $00 \cdot 51 \cdot 020.00506$. siniers du rôi donnaient à diner à tout le mon$\begin{array}{llllllllll}0 & 6 & .0 & 06 & 6 & 6 & 1 . \dot{2} 0 & 51.0\end{array}$

de, de manière que je ne sentais point la misè-

$\begin{array}{llllllllll}0 & 50 & 5 & .0 & .0 & 0 & 0 & 0 & i & .0\end{array}$

re. Nous couchions dans une garde-robe auprès

$$
\text { - } 0 \text {. }
$$

de la chambre du Due.

TBADUCCION INTERLINEAL.

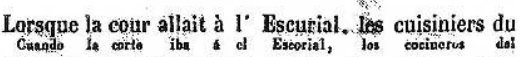
roi donnaieunt à diner à tout le monde, de manière que je

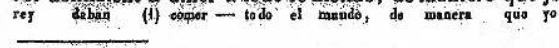

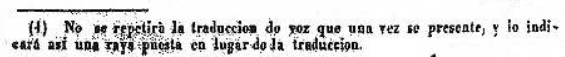


MENDIZÁBAL, Joaquín (1860)

Gramática francesa, $2^{\mathrm{e}}$ éd., Saragosse, Vicente Andrés

BIBLIOGRAPHIE

\section{Études}

ALONSO, Emilia, BRUÑA, Manuel, MUÑOZ, Maria éds. (1996) : La lingüística francesa : gramática, historia, epistemología. T. I-II. Seville, Grupo Andaluz de Pragmática.

BRUÑA, Manuel (2000) : «L'enseignement de la prononciation française aux Espagnols (XVI et XVII siècles) », in DE CLERCQ-LIOCE-SWIGGERS éds. (2000), 61-96.

BRUÑA, Manuel (2000) : «À nouveau sur l'enseignement de l'r français aux Espagnols depuis le XVII ${ }^{\mathrm{e}}$ siècle » in CASAL-CONDE-LAGO (2000), 177-201.

CASAL SILVA, Maria Luz, CONDE, Germán, LAGO, Jesús et alii éds. (2000) : La lingüística francesa en España camino del siglo XXI. Madrid, Arrecife.

CLERCQ, Jan de, LIOCE, Nico, SWIGGERS, Pierre éds. (2000) : Grammaire et enseignement du français 1500-1700. Louvain-Paris, Peeters.

ESCRICHE YMIEG, D.C.T. (1882) : « El método en la enseñanza de las lenguas. » BILE 140, 277-278.

HAMMAR, Elisabet éd. (1997) : Phonétique et pratiques de prononciation. L'apprentissage de la prononciation : chemin parcouru jusqu'à nos jours. Actes du Colloque de Linköping/Vadstena du 22 au 25 mai 1996. Documents pour l'histoire du français langue étrangère ou seconde 19. Paris, SIHFLES.

FISCHER, Denise (1997) : «L'enseignement de la phonétique française aux Espagnols, présenté dans les grammaires du $17^{\mathrm{e}}$ et $18^{\mathrm{e}}$ siècles. » Documents pour l'histoire du français langue étrangère ou seconde 19, 37-50. Paris, SIHFLES.

MILLET, Adrien (1933) : Les grammairiens et la phonétique ou l'enseignement des sons du français depuis le XVI e siècle jusqu'à nos jours. Paris, A. Maretheux et L. Pactat.

SALEMA, Maria-José (1997) : « L'apprentissage de la prononciation dans les lycées portugais avant $1910 »$, in HAMMARéd. (1997), 116-129.

SUPIOT, Alberto (1996) : « Descripción articulatoria y fonética correctiva en la tradición de la enseñanza del FLE en España », in ALONSO-BRUÑA-MUÑOZéds. (1996), II, 23-30.

\section{Sources premières}

ASCASO YPEREZ, Andrés (1854) : Tratado de Pronunciación francesa. Pampelune, Joaquín Lorda. $2^{\mathrm{e}}$ éd., 1876.

BERGNES DE LAS CASAS, Antonio (1845) : Novísimo Chantreau, o Gramática Francesa. Barcelone, Juan Oliveres. 
BORDE, P.B. (1888) : Método práctico de pronunciación francesa. Madrid, Capdeville. L'éd., 1886. BOUYNOT, Mauricio (1815) : Lecciones prácticas de lengua francesa. Valence, Manuel Muñoz. CASTAÑS, Átalo (s.d.) : Método de Ahn. Primer curso de francés. Segundo curso de francés. Madrid, Bailly-Baillière.

CORNELLAS, Clemente (1845) : Gramática francesa teórica-práctica para uso de españoles. Madrid, Viuda de Mayo.

COSTE, J. (1892) : Arte de hablar bien francés, en 8 días sin maestro. Barcelone, La Ilustración.

DELABORDE, R.E.L. (1859) : Nuevo Método de Lengua francesa en 80 días. Madrid, Mariano Saint de la Peña.

DUPUY, Pablo (1838) : Arte de hablar bien francés, o gramática completa, con un suplemento por P.N. Chantreau. Barcelone, Francisco Oliva.

ESCRICHE Y MIEG, D.C.T. (1881) : Ejercicios linguales de pronunciación francesa. Guadalajara, Establecimiento Tipográfico Provincial.

GIRAULT-DUVIVIER, Ch.-P. (1830) : Grammaire des Grammaires. Paris, Chez Janet et Cotelle. MENDIZABAL, Joaquín (1860) : Gramática francesa. $2^{\mathrm{e}}$ éd., Saragosse, Vicente Andrés.

MAC-VEIGH, Henry (1890): Método de Ahn. Primer curso de Francés, arreglado al castellano por el Profesor H. Mac-Veigh. Madrid, Bailly-Baillière. $\mathrm{I}^{\mathrm{e}}$ éd., 1857, Madrid, G. Hernández y Artés. NEVOT SANZ, S. (s.d.) : El francés al alcance de todos. Valence, Berenguer.

NOËL, M., CHAPSAL, M. (1849) : Abrégé de la grammaire française. $32^{\mathrm{e}}$ éd. Paris, Maire-Nyon.

PIFERRER, Francisco(1843) : Méthode pour apprendre en peu de jours à lire correctement le Français à l'usage de la jeunesse espagnole. Toulouse, Delsol.

PIFERRER, Francisco(1852): El idioma francés puesto al alcance de todos, o Método natural de aprender el francés. $3^{\mathrm{e}}$ éd. corrigée et augmentée. Madrid, Repullés. $1^{\mathrm{e} e ́ d . ~ 1846, ~ M a d r i d, ~ P a s a j e ~ d e ~ I r i s . ~}$

POU Y CLARA, J. (1886) : Método Pou. Sistema práctico para adquirir la pronunciación correcta del idioma francés. Barcelone, Imprenta española.

REINAUD, J.R. (s.d.) : Lengua francesa. Método Teórico-práctico. S.L.

SAUZEAU, Z. (1845) : Nueva Gramática de la lengua francesa. Madrid, Miguel de Burgos.

THIERS, V. (1841) : Alfabeto francés-español o Nuevo Método para aprender a leer el Francés en 6 lecciones. Valence, B. Monfort.

TRAMARRÍA Y CARRANZA, Francisco (1829) : Gramática francesa para uso de los españoles. Madrid, Aguado.

TRAUQUE, L. (1863) : Clave de lectura de la lengua francesa. Barcelone, V. Doménech.

\section{NOTES}

1. Voir la bibliographie à la fin de l'article.

2. Les références au Boletín de la Institución Libre de Enseñanza (BILE) ํo 140.1882 , correspondent à un compte rendu anonyme de l'article d'Escriche y Mieg qui y est fait dans la section « Revista de Pedagogía. » 
3. Nous traduirons généralement en français les propos des différents auteurs, sauf s'ils constituent une citation intéressante à reproduire telle quelle.

4. Ouvrage qu'il cite dans son Prologue, mais que nous n'avons pas réussi à consulter.

5. Par exemple, Reinaud cite les grammairiens français Napoléon Landais et Chapsal dans Tune de ses observations grammaticales (s.a.: 328 ).

6. «Este sonido suave y sordo, con-un ligero esfuerzo para sacarlo del pecho.»

7. «Del cuerpo de conocimientos que han de transmitirse al alumno."

8. Bien sûr, certains auteurs, tels Méndez Bejarano ou Araujo, ne vont pas faire la différence entre méthode scientifique et méthode pédagogique et vont proposer aux élèves débutants l'apprentissage de questions de grammaire historique, ou même d'ancien français !

9. «Solo he atendido aquí á sonidos, no á letras, puesto que mi objeto es puramente fonético. »

10. " no he creído inútil dar, para cada sonido y articulación desconocida, una ligera explicación de los órganos que en ellas toman parte, y disposición que afectan. »

11. « este sonido, fácil de producir, participa de la e y de la o española. De la primera toma la posición de la lengua, y de la segunda la de los labios. Para pronunciar esta vocal no hay más que disponer la lengua como si se tratara de hacer sonar una e, y, avanzando un poco los labios y redondeándolos cual si fuera á producirse una o, lanzar el aire.»

12. «Esta vocal participa de la i y de la u españolas. Tiene de la primera la posición de la lengua y de la segunda la de los labios. Para emitir este sonido basta preparar la lengua como si se trata de la i y poner los labios en la misma forma que si nos propusiésemos pronunciar nuestra u. »

13. «En cuanto a las reglas de pronunciación y modo de leer, no digo más que lo preciso, dexando lo que es de perfección al cuidado del buen maestro, que con pocas explicaciones de viva voz, enseña mucho más y mejor que todo lo que se pueda escribir, »

14. «Estos métodos olvidan á su vez que el aprendizaje de la lengua materna es lento, y que el extranjero que aprende sólo prácticamente otros idiomas, suele llegar á poseer un conocimiento muy superficial de ellos al cabo de ejercicio incesante. »

15. «Aprended un idioma extranjero como habeis aprendido vuestra lengua nativa : [...] Es el método de la naturaleza misma y el que emplea una madre cuando habla a su hijo, repitiéndole cien veces las mismas palabras, combinándolas imperceptiblemente y logrando de este manera hacerle hablar la lengua que ella habla. »

16. «Este método es simplemente de lectura francesa práctica, y por consiguiente sin reglas, porque no las necesita, si el profesor sabe y quiere cumplir su deber.»

17. «La lectura se hace en el testo numerado, comenzando el profesor, que lee todo el testo, y a continuación cinco, seis ó mas alumnos, hasta que la cátedra entera se halle empapada en la pronunciación. Será muy conveniente, para que no se debilite la sensación producida en el oido de los discípulos por el acento del profesor que éste vuelva á leer por lo menos después de cada dos alumnos. "

18. « en el estudio de una lengua viva se trata, no tanto de aprender teorías, como de crear un hábito de hablar al lado de otro ya existente, el de la lengua nativa : que se penetren de una verdad tan sencilla como trascendental, y es, que no se posee el francés hasta que se piensa en francés. »

19. «A la carrera."

20. «Recomiendo a éstos [a los alumnos] eficacísimamente que no pasen de un ejercicio á otro sin dominar el anterior : que no se cansen de repetirlos, aunque se figuren que están ya bastante enterados. "

21. " sencillo estudio en que se concede al entendimiento una buena parte de la turca que en Otros sistemas se encomienda esclusivamente á la memoria. »

22. « en lugar del cúmulo de reglas de pronunciación, ortografía, analogía y sintaxis, reglas cuya aplicación inmediata no ve el alumno, y que tienden constantemente á escaparse de la memoria 
[,..], [este sistema] le ofrece desde el principio hasta el fin una serle de ejercicios tan fáciles como sencillos. »

\section{RÉSUMÉS}

On peut distinguer quatre approches dans l'enseignement de la prononciation du français en Espagne au cours du XIXe siècle : certains auteurs reprennent l'essentiel de la méthode traditionnelle théorique, d'autres défendent une méthode théorico-pratique, selon divers degrés et modalités d'intégration, une troisième catégorie d'auteurs va proposer une approche méthodologique où la pratique joue un rôle essentiel, d'autres, enfin, inversent la place de la théorie et de la pratique et conçoivent des procédés totalement nouveaux d'enseignement de la prononciation.

We can identify four approaches in the teaching of the phonetics of French as a foreign language in Spain in the XIXth century : a first group of authors maintains the traditional theoretical method, a second group defend a " theoretical-practical method», where rules and explanations are completed by practice, a third group leans towards an approach where practical work is essential and where theory is banned; for the last group of authors practice comes first and theory afterwards. To do that, they have to invent new ways of teaching phonetics.

\section{INDEX}

Mots-clés : Enseignement de la prononciation, FLE, méthode traditionnelle, théorie, pratique, Espagne, XIXe siècle

Keywords : Teaching of the phonetics, French as a foreign language, traditional theoretical method, theory, practice, Spain, XIXth century

\section{AUTEUR}

\section{MARIA EUGENIA FERNÁNDEZ FRAILE}

Université de Grenade 